\title{
Delayed Duodenal Perforation of an Endoscopic Mucosal Resection- Induced Ulcer due to a Foreign Body
}

\author{
Tae-Geun Gweon, Cheal Wung Huh and Byung-Wook Kim \\ Division of Gastroenterology, Department of Internal Medicine, College of Medicine, Incheon St. Mary's Hospital, The Catholic University of \\ Korea, Seoul, Korea
}

A 79-year-old man was referred to our hospital for removal of an adenoma in the afferent loop of a Billroth II anastomosis. Thirty-nine years earlier, he had undergone subtotal gastrectomy with Billroth II anastomosis because of gastric ulcer bleeding. His laboratory findings were unremarkable. Esophagogastroduodenoscopy revealed a subtly yellowish, elevated lesion $10 \mathrm{~mm}$ in diameter in the afferent loop of the Billroth II anastomosis. (Fig. 1A) The polyp was located in the distal portion approximately 3-4 cm apart from the anastomosis site. We resected the polyp by endoscopic mucosal resection (EMR), and the resection site was clear (Fig. 1B). No immediate adverse event related to the procedure was observed. After 2 days, however, the patient visited the emergency department because of severe epigastric pain. Abdominal computed tomography revealed fluid collection and free air around the afferent loop and right colon (Fig. 2A). Esophagogastroduodenoscopy revealed a sharp foreign body penetrating the EMR site (Fig. 2B). We meticulously examined the penetrating site using grasper forceps and found that the foreign body was directly penetrating the EMR-induced ulcer (Fig. 2C). We removed the foreign body using grasper forceps and closed the perforation site with endoclips (EZ Clip; Olympus, Tokyo, Japan), using single closure methods. The foreign body was a

Received: April 11, 2019 Revised: May 17, 2019

Accepted: May 21, 2019

Correspondence: Cheal Wung Huh

Division of Gastroenterology, Department of Internal Medicine, College of Medicine, Incheon St. Mary's Hospital, The Catholic University of Korea, 56 Dongsu-ro, Bupyeong-gu, Incheon 21431, Korea

Tel: +82-32-280-5052, Fax: +82-32-280-5987, E-mail: huhcw@catholic.ac.kr ORCID: https://orcid.org/0000-0001-7327-8503

(c) This is an Open Access article distributed under the terms of the Creative Commons Attribution Non-Commercial License (http://creativecommons.org/ licenses/by-nc/3.0) which permits unrestricted non-commercial use, distribution, and reproduction in any medium, provided the original work is properly cited. suture material used in the previous gastric surgery (Fig. 2D). The patient received conservative medical treatment without surgery. At 6-week follow-up, he had recovered completely without any symptoms.

Endoscopic resection is becoming the first choice of treatment for superficial duodenal epithelial neoplasia., ${ }^{1,2}$ However, exposure to pancreatic juice or bile, thin duodenal wall, and retroperitoneal location increase the risk of perforation. ${ }^{3}$ To prevent delayed perforation, complete closure of the mucosal defect after endoscopic resection is proposed, with favorable clinical outcomes. ${ }^{4}$ We report a rare case in which a suture material used in the previous surgery caused delayed perforation of the EMR site. Complete closure of the mucosal defect after endoscopic resection is required to prevent delayed complications, even in the case of small duodenal polyps.

\section{Conflicts of interest}

The authors have no financial conflicts of interest.

\section{REFERENCES}

1. Yahagi N, Kato M, Ochiai Y, et al. Outcomes of endoscopic resection for superficial duodenal epithelial neoplasia. Gastrointest Endosc 2018;88:676-682.

2. Tomizawa Y, Ginsberg GG. Clinical outcome of EMR of sporadic, nonampullary, duodenal adenomas: a 10-year retrospective. Gastrointest Endosc 2018;87:1270-1278,

3. Ochiai Y, Kato M, Kiguchi Y, et al. Current status and challenges of endoscopic treatments for duodenal tumors. Digestion 2019;99:21-26.

4. Kato M, Ochiai Y, Fukuhara S, et al. Clinical impact of closure of the mucosal defect after duodenal endoscopic submucosal dissection. Gastrointest Endosc 2019;89:87-93. 


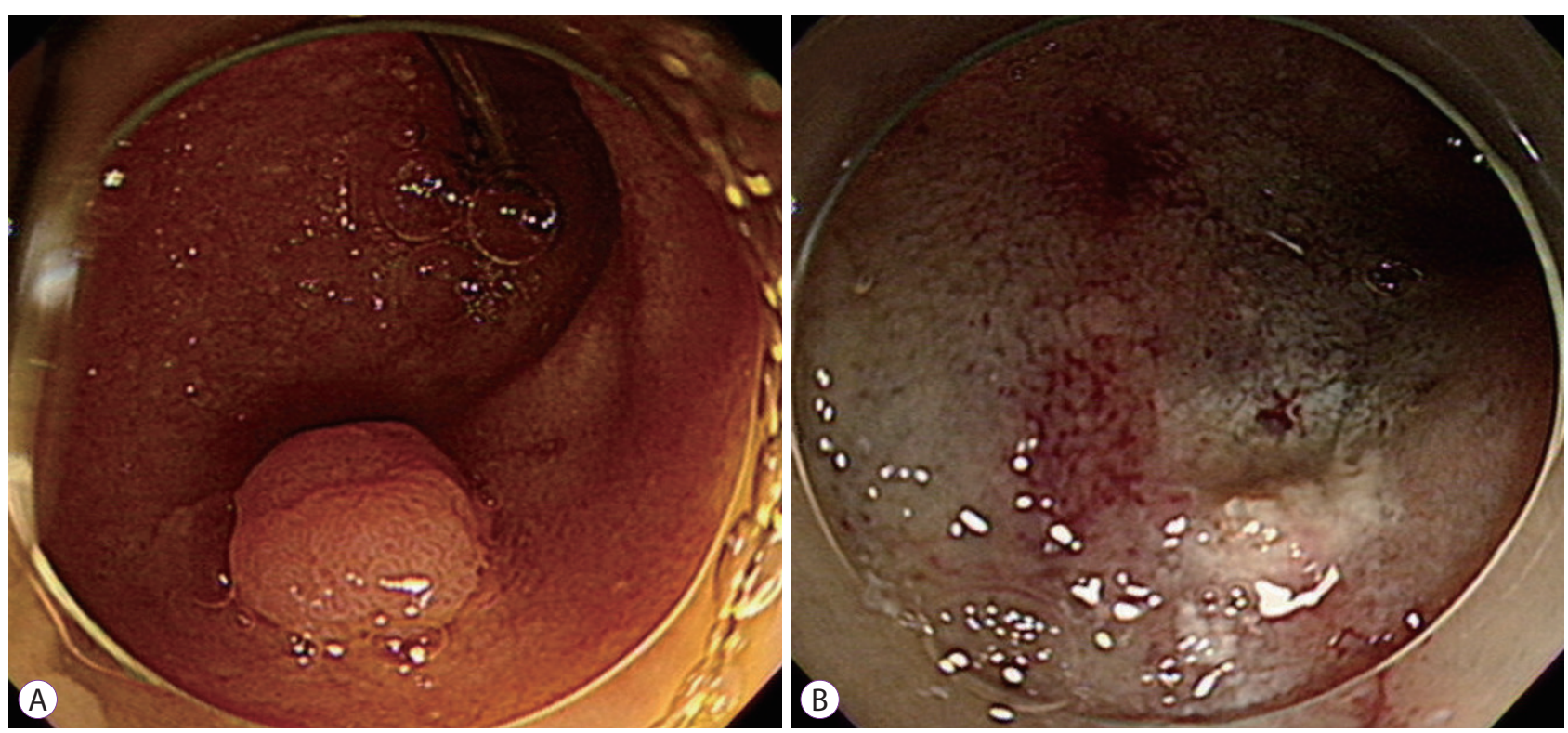

Fig. 1. (A) An esophagogastroduodenoscopy image of a subtly yellowish, elevated lesion $10 \mathrm{~mm}$ in diameter in the afferent loop of a Billroth II anastomosis. (B) Clear resection site after endoscopic mucosal resection.
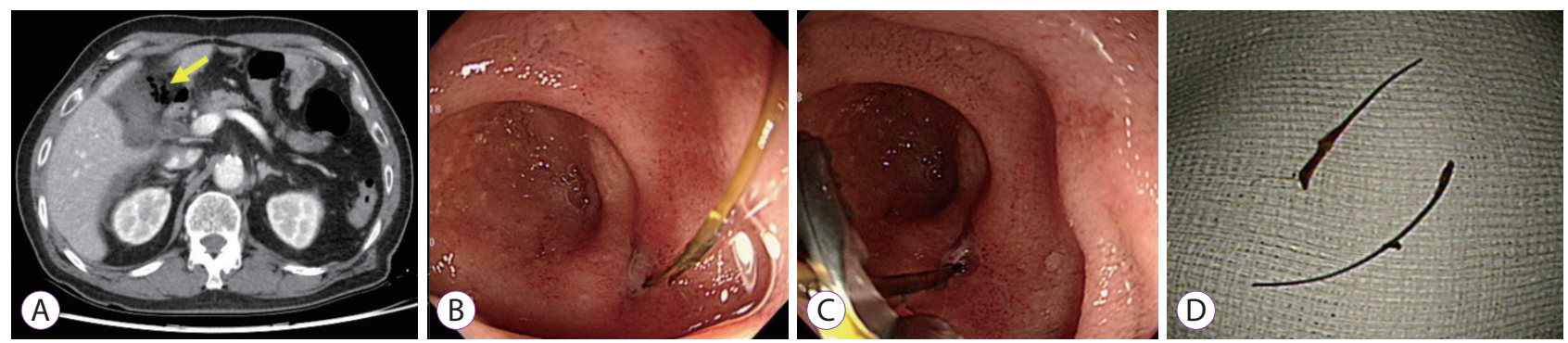

Fig. 2. Images after 2 days. (A) A computed tomography scan showing fluid collection and free air around the afferent loop and right colon (yellow arrow). (B, C) An esophagogastroduodenoscopy image showing a sharp foreign body penetrating the endoscopic mucosal resection site. (D) The foreign body is a suture material used in the previous gastric surgery. 